\title{
Modern Radio Universe
}

\section{Philip Diamond ${ }^{1}$}

Director Jodrell Bank Centre for Astrophysics and Chairman of the SOC,

Jodrell Bank Centre for Astrophysics, The University of Manchester, Oxford Road, Manchester, M13 9PL $U K$

E-mail: pdiamondejb.man.ac.uk

\section{Richard Schilizzi ${ }^{2}$}

Director SKA Program Development Office and co-Chairman of the SOC,

SKA Project Office, Jodrell Bank Centre for Astrophysics, The University of Manchester, Oxford Road, Manchester, M13 9PL UK

E-mail: schilizzileskatelescope.org

The Modern Radio Universe conference drew 220 participants from 23 countries to the University of Manchester in October 2007 to discuss the current state of knowledge, as revealed by observations and theory, of the key science themes to be addressed by the Square Kilometre Array (SKA). In this contribution we present a short overview of this conference, including both the scientific and historical motivation and the new science presented.

From planets to dark energy: the modern radio universe University of Manchester, Manchester, UK

1-5 October, 20

\footnotetext{
$1 \quad$ Speaker
} 


\section{Introduction}

The Modern Radio Universe conference drew 220 participants from 23 countries to the University of Manchester in October 2007 to discuss the current state of knowledge, as revealed by observations and theory, of the key science themes to be addressed by the Square Kilometre Array (SKA). The SKA will be the largest development in radio astronomy during the coming decade. However, the SKA and other next generation radio telescopes build upon a long history of developments in radio astronomy. This meeting was timed to celebrate this history whilst looking to the future. October 4th 2007 was the 50th anniversary of the launch of Sputnik I and the radar detection of the Sputnik launch rocket by the 76-m (250-ft) Lovell telescope at Jodrell Bank Observatory. Hence, this conference celebrated 50 years of the Space Age and the Lovell telescope by reviewing the state of modern radio astronomy and the new scientific horizons that will be opened by the next generation of radio telescopes and in particular the SKA.

The conference began with an overview of the corner-stone facilities that will be available to astronomers in the coming years, facilities such as ALMA, JWST, EVLA, e-MERLIN, LOFAR, ELT and others and, of course, the SKA. This set the scene for the talks and posters that followed. The SOC split the programme into 5 main themes, based upon the SKA's Key Science Projects; they asked invited speakers to describe the current state of knowledge of a particular area, to discuss how the SKA might advance that area and also, where appropriate, to put the science into context bearing in mind the wonderful facilities which will be available in the future.

The five themes upon which the conference focused were:

- The evolution of galaxies and cosmic large scale structure

- Probing the Dark Ages - the first black holes and stars

- Strong field tests of gravity using pulsars and black holes

- The origin and evolution of Cosmic Magnetism

- The Cradle of life

In all there were 74 presentations and 64 posters covering these areas of science.

On $4^{\text {th }}$ October, the anniversary of Sputnik, the SOC had wisely decided that the morning would be a poster viewing session (the conference dinner had occurred on the previous evening) and so after coffee all participants were transported $20 \mathrm{~km}$ south of Manchester to Jodrell Bank Observatory. There a celebration of the historic events of 50 years ago passed off well under sunny skies and in the presence of the Minister for Science and Innovation, Ian Pearson; His Excellency the Ambassador of the Russian Federation, Yuri Fedotov; the President of the Royal Society, Lord Rees of Ludlow; the President and Vice-Chancellor of the University of Manchester, Prof. Alan Gilbert and last, but most definitely not least, Prof. Sir Bernard Lovell. The highlight of the day was a tour of the telescope, of which more than 180 people took advantage.

Prof. Philip Diamond (Director Jodrell Bank Centre for Astrophysics and Chairman of the SOC) Prof Richard Schilizzi (Director SKA Program Development Office and co-Chairman of the SOC) 